\title{
On the Concept of Meaningfulness in Constrained Type-2 Fuzzy Sets
}

\author{
Pasquale D'Alterio, Jonathan M. Garibaldi and Robert John \\ School of Computer Science \\ University of Nottingham \\ Nottingham, UK \\ \{pasquale.d'alterio, jon.garibaldi, robert.john\}@ nottingham.ac.uk
}

\begin{abstract}
Constrained type-2 fuzzy sets have been proposed as a tool to model type-2 fuzzy sets starting from a type-1 generator set with uncertainty. This constrained representation only defines as acceptable the embedded sets that have the same shape as the generator set in order to process only membership functions that are considered "meaningful" when using fuzzy operators such as centroid defuzzification. However, the idea of "meaningfulness" has never been clearly defined; at the same time there are some contexts in which a given concept (e.g. medium height) can be reasonably represented by multiple shapes, such as triangular and Gaussian. The aim of this paper is both to formally define the idea of meaningfulness of shapes and to extend the formal definitions of constrained interval type-2 fuzzy sets in order to allow the presence of multiple shapes among the acceptable embedded sets.
\end{abstract}

Index Terms-constrained type-2, type-2 fuzzy sets, embedded sets, meaningful membership function

\section{INTRODUCTION}

Since their introduction, type-2 fuzzy sets (T2 FS) [1] have been widely used due to their inherent capability of modeling uncertainty with membership functions. Some of the most widely used fuzzy operators, such as Karnik-Mendel (KM) centroid defuzzification [2], make of use of the concept of embedded sets (ES). They can be intuitively seen as a path along the T2 surface of the FS they belong to; additionally, it has been proven [1] that any T2 FS can be represented as the union of all its ESs (representation theorem).

In the past few years, however, some research papers [3]-[5] argued that in some contexts (e.g. human reasoning) the current formal definition of T2 FSs gives too much "mathematical freedom" in the identification of the boundaries of the footprint of uncertainty (FOU) and its ESs. As a result, the semantic relation between the T2 FS and the concept it should model is lost.

For this reason constrained type-2 fuzzy sets (CT2 FSs) were introduced [6]. They identify a subset of T2 FSs that can be represented by the use of only "meaningful" membership functions (MF), constraining both the possible shapes of the ESs and the FOU boundaries. The main idea behind them, is to represent T2 FSs as T1 FSs with uncertainty on their exact location on the x-axis. Therefore, the ESs that are considered acceptable (acceptable embedded sets, AES) are obtained by the translation on the $\mathrm{x}$-axis of the generator set (GS) while the FOU is obtained as the union of all the AES.

So far, however, no clear definition has been given of what a "meaningful" shape is in the context of CIT2 FSs. At the same time, it has been analyzed in [7] that operations on CT2 may produce T2 FSs that formally are not CT2 FSs, i.e. it is not possible to find a GS that would fully cover their FOU. That is because all the AESs obtained from the fuzzy operators have different shapes, regardless of the fact that they could all be reasonable for the operation result they represent. The aim of this paper is to both clarify the concept of "meaningfulness" in CIT2 FSs and to extend the original CT2 definitions, in order to include different and more general constraints that go behind the requirement of having ESs with the same shape. By doing this, we will provide a more powerful modeling tool which can be useful in all the cases where different shapes (e.g. both triangular and Gaussian) are considered acceptable for the representation of a given concept.

\section{Preliminary Definitions}

This section will be focused on basic fuzzy logic definitions that will be used in the rest of the paper. They are taken or rephrased from [1], [6], [8].

Definition 1. A type-1 fuzzy set (T1 FS), denoted A, is characterized by a T1 membership function $(M F) \mu_{A}: X \mapsto[0,1]$, i.e.,

$$
A=\left\{\left(x, \mu_{A}(x)\right) \mid x \in X\right\}
$$

with $X$ being the universe of discourse (UOD).

Definition 2. A type-2 fuzzy set (T2 FS), denoted $\tilde{A}$, is characterized by a T2 MF $\mu_{\tilde{A}}: X \times J_{x} \mapsto[0,1]$, where $J_{x} \subseteq[0,1]$, i.e.,

$$
\tilde{A}=\left\{\left((x, u), \mu_{\tilde{A}}(x, u)\right) \mid x \in X, u \in J_{x} \subseteq[0,1]\right\}
$$

in which $X$ is the UOD. $\tilde{A}$ can also be expressed as:

$$
\tilde{A}=\int_{x \in X} \int_{u \in J_{x}} \mu_{\tilde{A}}(x, u) /(x, u) \quad J_{x} \subseteq[0,1]
$$

Definition 3. An interval type-2 fuzzy set (IT2 FS), denoted $\tilde{A}$, is characterized by an IT2 MF $\mu_{\tilde{A}}: X \times J_{x} \mapsto\{0,1\}$, 
where $J_{x} \subseteq[0,1]$, i.e.,

$$
\tilde{A}=\left\{\left((x, u), \mu_{\tilde{A}}(x, u)\right) \mid x \in X, u \in J_{x} \subseteq[0,1]\right\}
$$

in which $X$ is the UOD. $\tilde{A}$ can also be expressed as:

$$
\tilde{A}=\int_{x \in X} \int_{u \in J_{x}} 1 /(x, u) \quad J_{x} \subseteq[0,1]
$$

Definition 4. Give a T2 FS $\tilde{A}$, its footprint of uncertainty (FOU) [9] is the set of points $(x, u)$ for which $\mu_{\tilde{A}}(x, u)>0$ :

$$
\operatorname{FOU}(\tilde{A})=\left\{(x, u) \mid(x, u) \in X \times[0,1], \mu_{\tilde{A}}(x, u)>0\right\}
$$

Definition 5. At each value of $x$, say $x=x^{\prime}$, the 2-D plane whose axes are $u$ and $\mu_{\tilde{A}}\left(x^{\prime}, u\right)$ is called a vertical slice of $\mu_{\tilde{A}}\left(x^{\prime}, u\right)$. A secondary $M F$ is a vertical slice of $\mu_{\tilde{A}}(x, u) . I t$ is $\mu_{\tilde{A}}\left(x=x^{\prime}, u\right)$ for $x \in X$ and $\forall u \in J_{x^{\prime}} \subseteq[0,1]$, i.e.,

$$
\mu_{\tilde{A}}\left(x=x^{\prime}, u\right) \equiv \mu_{\tilde{A}}\left(x^{\prime}\right)=\int_{u \in J_{x^{\prime}}} f_{x^{\prime}}(u) / u \quad J_{x^{\prime}} \subseteq[0,1]
$$

Definition 6. The domain of a secondary MF is called the primary membership of $x$. In (2), $J_{x}$ is the primary membership of $x$.

Definition 7. A type-2 embedded set (T2 ES), denoted $\tilde{A}_{E}$, is a path along the $T 2$ set it belongs to. It contains only one primary degree $u_{x}$ for each $x$, with its associated secondary grade $v_{x}$ :

$$
\mu_{\tilde{A}_{E}}\left(x, u_{x}\right)=v_{x} \quad x \in X, u_{x} \in J_{x}
$$

Definition 8. A type-1 embedded set (T1 ES), denoted $A_{E}$ represents a projection of a T2 ES, i.e. its secondary degree has been dropped. Therefore it contains one primary degree $u_{x}$ for each $x$ :

$$
\mu_{A_{E}}(x)=u_{x} ; \mu_{A_{E}}\left(x, u_{x}\right)=v_{x} \quad x \in X, u_{x} \in J_{x}
$$

\section{Constrained Interval Type-2: Current Formal DEFINITIONS}

In the remainder of the paper, for simplicity, we will focus on constrained interval type-2 (CIT2) FSs rather than the more general CT2 representation. CIT2 FSs are defined starting from a T1 FS, called generator set (GS), which is the underlying T1 MF with uncertainty modeling a given concept. To obtain the AESs of a CIT2 FS, we translate our GS along the X-axis using a set of real values. This set is called the displacement set:

Definition 9. A displacement set (DS), denoted $D$, is a closed set of real numbers such that:

$$
D \subseteq \mathbb{R}, 0 \in D
$$

With the DS and the GS, we can generate the set of AESs that share the same shape:

Definition 10. A collection of T1 acceptable embedded sets (CAES), is a set of T1 FSs obtained from the shifting of a T1
$G S G$. Formally, each of the acceptable embedded sets (AES) $S$ in a CAES can be expressed as:

$$
S=\left\{\left(x, \mu_{S}(x)\right) \mid x \in X\right\}
$$

where

$$
\mu_{S}: X \mapsto[0,1], \exists c \in D: \mu_{S}(x)=\mu_{G}(x-c), \forall x \in X
$$

given a UOD X, a DS D, a T1 GS G.

The CAES is then used to define a CIT2 FS:

Definition 11. A constrained interval type-2 fuzzy set (CIT2 FS) $\breve{A}$, is characterized by an IT2 MF $\mu_{\breve{A}}$ with $J_{x}=$ $\bigcup_{S \in C A E S_{\breve{A}}} \mu_{S}(x)$ :

$$
\breve{A}=\left\{\left((x, u),\left(\mu_{\breve{A}}(x, u)\right)\right) \mid x \in X, u \in \bigcup_{S \in C A E S_{\breve{A}}} \mu_{S}(x)\right\}
$$

with CAES $_{\breve{A}}$ being a CAES. $\breve{A}$ can also be expressed as:

$$
\begin{aligned}
\breve{A} & =\int_{x \in X} \int_{u \in J_{x}} 1 /(x, u) \\
& =\int_{x \in X} \int_{u \in \bigcup_{S \in C A E S_{\breve{A}}} \mu_{S}(x)} 1 /(x, u)
\end{aligned}
$$

As a result of these definitions, the CIT2 FSs are all and only the IT2 FS with a FOU that is obtainable as the union of ESs sharing the same shape (i.e. the one determined by the GS). By doing this, we make sure that all the fuzzy operators (e.g. centroid defuzzification) only process ESs with a "meaningful" shape.

The specific meaning of "meaningfulness", however, has not been defined clearly and the identification of the correct shape for the GS and the AESs is left to the fuzzy system designer. Additionally, Def. 10 poses a restriction that can be excessive in contexts in which more than one shape is reasonable to model a given concept, as will be analysed in the next section.

\section{CiT2 Limitations: Two CASE STUdies}

Even though the idea behind CT2 FSs was to provide a representation that keeps a "meaningful" relation between T2 FSs and the concept they model, there are some cases in which the restriction of having only AESs sharing the same shape is too limiting. We will support this claim by providing two practical example of CIT2 application in which the use of different shapes is needed to obtain an accurate representation of the modeled scenario.

\section{A. Modeling Words}

In this thought experiment, our goal is to obtain a CIT2 FS for the concept of medium height. To do that, we start from a Gaussian T1 as our GS (Fig. 1) to which we want to add uncertainty.

In order to obtain our CAES, we asked different people to place our T1 GS on the x-axis (similar approaches can be found in [10], [11]). Since the concept of medium height 


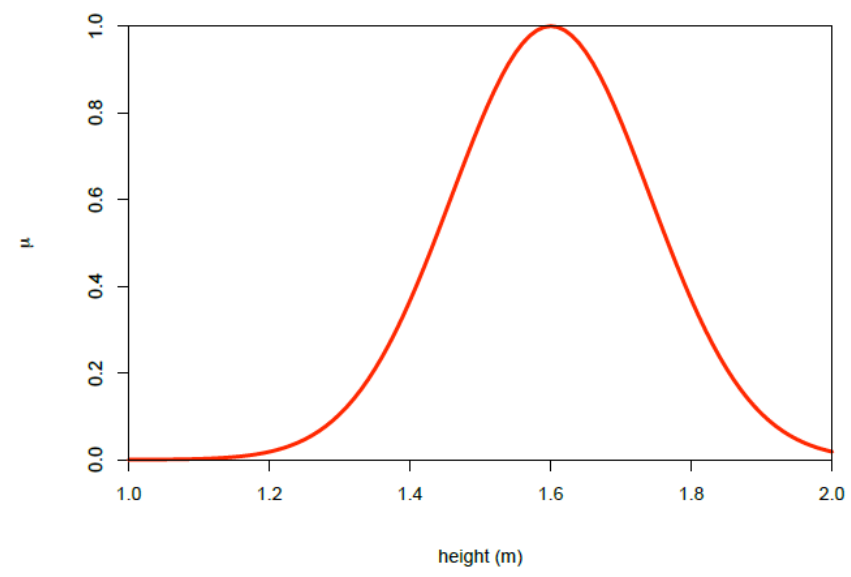

Fig. 1. T1 GS modeling medium height (picture from [6])

varies slightly from person to person, it is likely that we would obtain something similar to what is shown in Fig. 2.

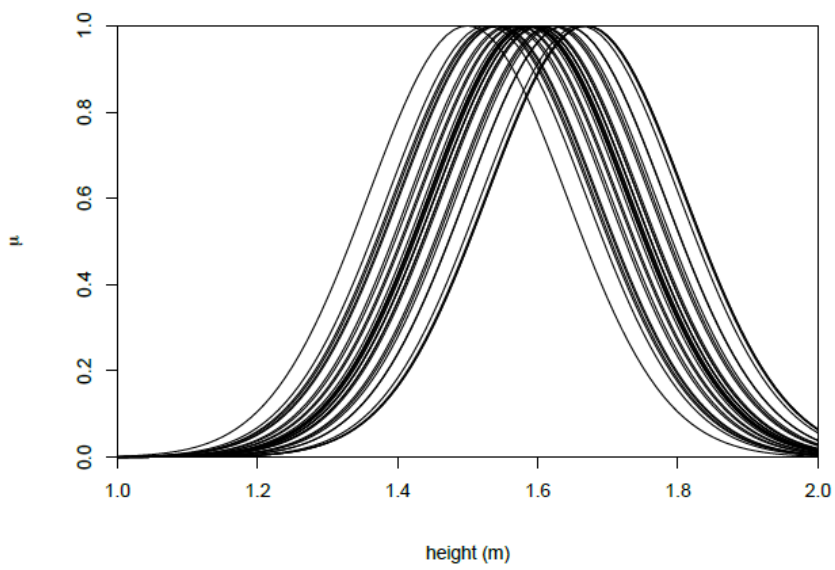

Fig. 2. AES obtained from the medium height experiment (picture from [6])

By using the approach described above, we are making sure that only the ESs with a "meaningful" shape are included in the AES and then processed by fuzzy operators such as KM centroid defuzzification. Specifically, all the AES keep a semantic relation with the concept of medium height they are modeling.

\section{B. Analysis - I}

We can see how the idea of imposing the use of one specific Gaussian for the generation of the CAES is very limiting in this case. For example, one could imagine that some of the participants would want to change the spread of the Gaussian or would want to use triangular shapes instead of Gaussian ones. This would be unacceptable by the current CIT2 definitions since a CAES with different shapes would not satisfy Def. 10. Nevertheless, in this example there are multiple shapes that can be considered "meaningful", in the sense that they keep the semantic relation with the concept they model. In this case, we can see that the concept of "meaningfulness" is not kept by one specific shape but it is rather the result of the satisfaction of a set of constraints that we implicitly impose on words in human reasoning. For example, one could imagine that in the case of "medium height", the meaningfulness and the semantic relation is kept by all the symmetric shapes that are monotonically increasing up to a plateau and then monotonically decreasing.

The analysis of this experiment suggests that the idea of imposing one shape to all the ESs is only one of the possible constraints that a designer would want to use for a T2 FS and that the concept of "meaningfulness" is not related to one specific shape but is rather the result of the satisfaction of a set of implicit constraints that are related to the concept we are working with. Furthermore, even non-convexity and normality, which are usually described as "desirable properties" for MFs [3], [5], [6], can be "non-meaningful" in some contexts, as shown in the next subsection.

\section{Fuzzy system outputs: non-normal and convex membership functions}

To show that non-normal and/or convex MFs can still be meaningful, we will analyze the problem that was described in [7] in relation to fuzzy outputs of CIT2 fuzzy rules, i.e. fuzzy rules in which all the antecedent and consequent FSs involved are CIT2 FSs. Consider the following CIT2 fuzzy rule $R$ :

$$
R \text { : IF } x_{1} \text { is } \breve{A} \text { AND } x_{2} \text { is } \breve{B} \text { THEN } y \text { is } \breve{C}
$$

The consequent FS $\breve{C}$ is shown in Fig. 3. It is obtained using a triangular MF as a GS and a discrete DS to generate the AESs.

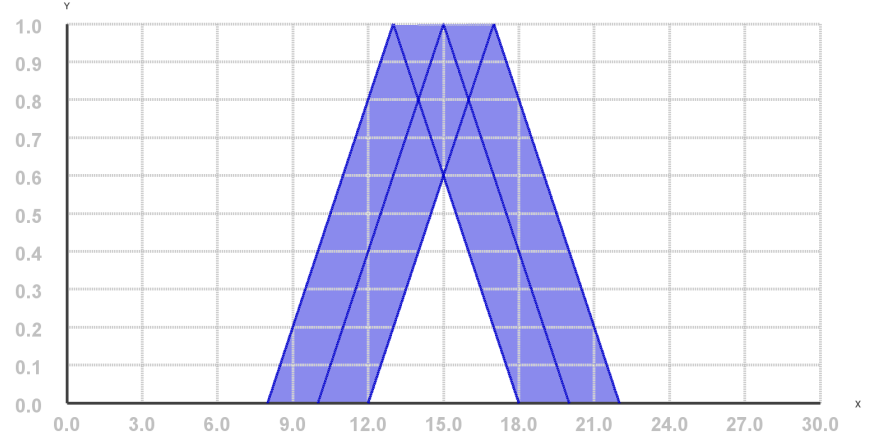

Fig. 3. Consequent CIT2 FS $\breve{C}$ used in the rule $R$ (FOU in light blue)

To carry out the inference in a rule, we will use the process described in [7]: the CIT2 fuzzy rule is expanded in a set of T1 fuzzy rule; each one of them is obtained by substituting the CIT2 FSs involved in the rule with one of their T1 AESs. The goal of the process is to obtain the AESs of the FS resulting from the rule evaluation.

The fuzzy rule output shown in Fig. 4 has been obtained using the process described above, with the minimum function for the conjunction (and) and implication operator. 


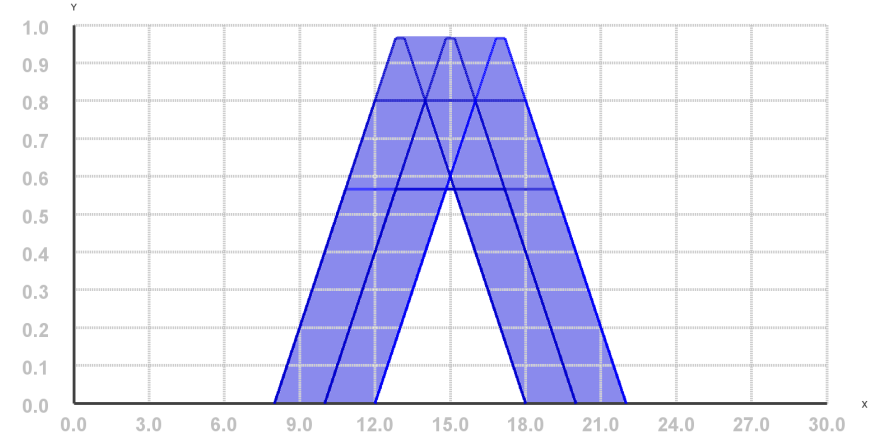

Fig. 4. CIT2 output from the inference of a CIT2 rule in which all the sets involved are fixed-shape CIT2 sets (FOU in light blue)

\section{Analysis - II}

It is clear that the collection of T1 ES in Fig.4 is not a CAES as defined in Def. 10: since they have been obtained from the same triangular shape truncated at different height, it is not possible to identify a valid T1 GS. In other words, it is not possible to choose one of the T1 FS in Fig. 4 as a GS, so that the other AESs could be obtained from the translation along the $\mathrm{x}$-axis of the GS. Furthermore, these AESs are nonnormal. Therefore, we can conclude that the fuzzy result of a CIT2 fuzzy rule is not a CIT2 FS, according to Def. 14. This seems to suggest that the collection of T1 FSs in Fig. 4 is not "meaningful" in our context.

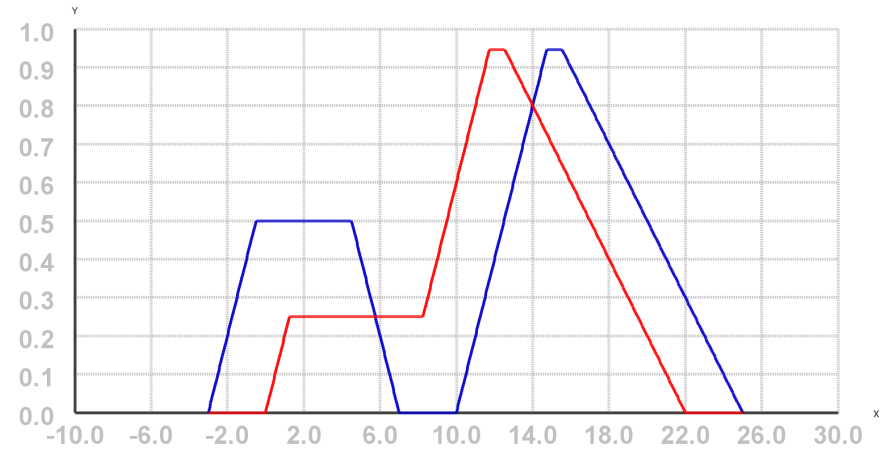

Fig. 5. Examples of two AES obtainable from a CIT2 Mamdani fuzzy system

However, each of those T1 FSs represents a plausible T1 fuzzy rule output since they have been obtained as results of T1 fuzzy rules by picking one of the AESs of each CIT2 FSs involved in the CIT2 rule. Intuitively, the T1 FSs in Fig. 4 represent possible T1 fuzzy rule outputs when the uncertainty modeled around the T1 GS is removed and the CIT2 FS collapses to one of its AESs, i.e. when the one of the possible locations of each T1 GS on the x-axis is chosen. Therefore, the collection of T1 FSs in Fig. 4 represents all the possible T1 fuzzy outputs that can be obtained by taking into account the uncertainty on the T1 GSs of all the CIT2 FSs involved in the rule.

This analysis supports the fact that the FSs in Fig. 4, still carry a "meaningful" connection when it comes to the representation of fuzzy rule outputs even though they do not satisfy the definition of CAES as described in Def. 10 and are non-normal T1 FSs. In contrast, the standard IT2 representation would consider as acceptable all the ESs of a given IT2 fuzzy rule output, regardless of the fact that they could or could not represent an actual T1 rule output FS.

In addition to that, if we have a Mamdani fuzzy system with multiple CIT2 fuzzy rules that we combine by the union operator, we can obtain a collection of T1 FSs which is non-convex (Fig. 5). For the same reasons discussed above, however, those FSs would still be "meaningful" for the representation of a Mamdani system output since they represent plausible T1 system outputs when an exact location for all the GSs is chosen. We can therefore conclude that even non-convexity and normality, in contrast to what has been initially stated in the original definition of CIT2 FSs [6], can be acceptable properties with a clear "meaning" (i.e. represent T1 fuzzy rule outputs).

\section{EXtending Constrained Type 2 Fuzzy Sets}

As a result of the analysis carried out in the previous section, we believe that new formal definitions for CIT2 FSs are needed. As already discussed, the original concept of "meaningfulness" fulfilled by the use of a single shape for all the ESs can be limiting in some contexts. Specifically, it is only useful when the kind of uncertainty we are modeling is restricted to the exact location of the T1 GS on the X-axis. In addition to that, it is not clear when and why a shape is considered to be meaningful in a given scenario. For these reasons, we believe that a formalization of the concept of meaningfulness is needed. Specifically, we propose a novel representation of the CAES (Def. 10) based on the satisfaction of a set of constraints. This approach both formalises the concept of "meaningfulness" into the satisfaction of constraints and provides a representation that makes ESs with different shapes acceptable.

Definition 12. A collection of $T 1$ acceptable embedded sets (CAES), is a set of T1 FSs satisfying a set of $n$ constraints constraints $C_{1}, \ldots, C_{n}$ :

$$
C A E S=\left\{S \mid \mu_{S}: X \mapsto[0,1], C_{1}(S) \star \ldots \star C_{n}(S)\right\}
$$

with $X$ being the UOD and each of the $\star$ being either $\wedge$ or $\vee$.

This new definition of CAES can then be used in Def. 11 to obtain our new CIT2 FSs. All the other definitions remain unchanged.

In the context of human reasoning, those constraints are implicitly imposed by people on the words they use. For example, as discussed in Sec. IV, when using words such as medium, one can expect the MF modeling this concept to be monotonically increasing-decreasing, symmetric and non-convex. In other scenarios, MFs are obtained from data analysis, as in [4]. In this case, the constraints are given by empirical or theoretical relations between the values of the universe of discourse. The original idea of constraining the ESs 
to share the same shape is only one of the possible constraints we may want to impose on the T2 FS we are modeling.

To prove that this new formulation is more general than the old one, we will show that any CAES that satisfies Def. 10, can also be obtained by the use of constrains as in Def. 12 . Specifically, given a CAES $A$ where all its T1 sets are obtained from a T1 GS $G$ and a DS $D, A$ can also be expressed as:

$$
A=\left\{S \mid \mu_{S}: X \mapsto[0,1], C_{1}(S)\right\}
$$

where:

$$
C_{1}(S)= \begin{cases}\text { true } & \text { if } \exists c \in D: \mu_{S}(x)=\mu_{G}(x-c), \forall x \in X \\ \text { false } & \text { otherwise }\end{cases}
$$

\section{A. Applications}

To show a practical application of this new definition of CIT2, we will analyze a case that is very similar to the one presented in the first part of Sec. IV. Just like in the other thought experiment, we want to model a CIT2 FS representing medium height starting from T1 MFs obtained from a survey. The difference is that, this time, each person can freely choose the shape that he or she considers to be the most appropriate for this context. A possible experimental result is shown in Fig. 6. Since we would likely obtain different MFs (e.g. triangular and Gaussian), this scenario couldn't be modeled with the old CIT2 definition. However, both triangles and Gaussians are appropriate in this context.

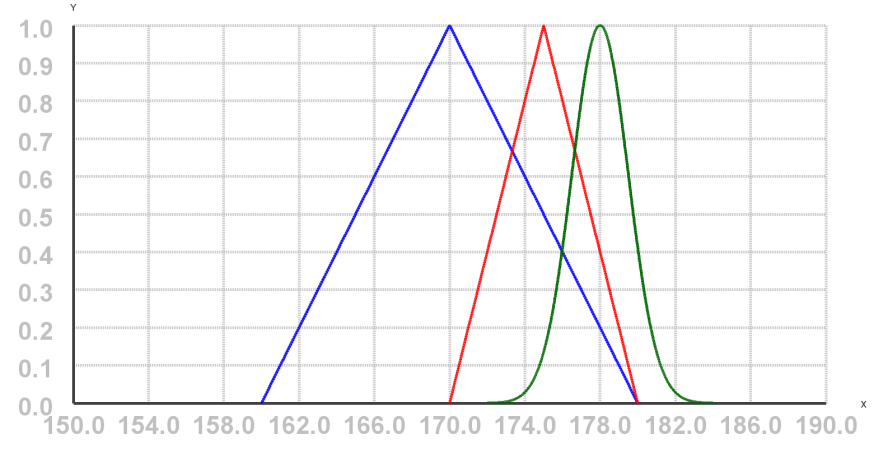

Fig. 6. Possible T1 MFs modeling medium height

When the number of AES is finite and obtained from surveys or data analysis, generating the constraints for our CAES is trivial. One strategy would be to put these MFs in a set named $E$ and then define the following constraint $C_{1}$ :

$$
C_{1}(S)= \begin{cases}\text { true } & S \in E \\ \text { false } & \text { otherwise }\end{cases}
$$

This constraint can then be used to build a CAES and a CIT2 FS as in Def. 11.

The idea of defining a CAES as T1 MFs satisfying a set of constraints is more powerful when the number of shapes that are acceptable is infinite. For example, we may want to consider as acceptable for medium height all the Gaussians having mean between 170 and 180 and having a standard deviation between 1 and 1.5. This scenario can be easily modeled by the following constraint:

$$
C_{G}(S)= \begin{cases}\text { true } & \exists \mu, \sigma: 170 \leq \mu \leq 180,1 \leq \sigma \leq 1.5 \\ & \mu_{S}(x)=\frac{1}{\sigma \sqrt{2 \pi}} e^{-(x-\mu)^{2} / 2 \sigma^{2}}, \forall x \in X \\ \text { false } & \text { otherwise }\end{cases}
$$

Even if all the MFs satisfying the constraints $C_{G}$ are Gaussians, it would have not been possible to model this scenario using the old CIT2 definition. That is because the difference in their variance could not by modeled by Def. 10 since it considers only as acceptable Gaussians that differed for their mean, i.e. Gaussians that be obtained as the translation along the $\mathrm{x}$-axis of a GS.

\section{DISCUSSION}

The new CIT2 definition, based on constraints satisfaction, allows us to model a broader set of scenarios, like the ones described in Sec. IV, V. In addition to that, no property is imposed a priori on our sets, not even normality or nonconvexity.

This represent a significant difference when compared to the other constrained approach introduced by Wu in [3], where any "well shaped" [5] IT2 FS is represented using only normal and non-convex ESs.

In contrast, we believe that the imposition of normality and non-convexity can be too restrictive in some cases and not sufficient in others. For example, when working with fuzzy outputs these properties are usually not necessary since these sets keep their own interpretability regardless of their convex o non-normal shape.

If, instead, we only want one or a limited set of specific shapes to be acceptable for our ESs, normality and nonconvexity alone are not sufficient to guarantee that our T2 FSs will keep a semantic meaning with the concept they're modeling.

In addition to that, as already analyzed in [5], Wu's approach has the downside of being unusable in Mamdani systems since there is no guarantee that its fuzzy output will maintain the "well shaped" properties when rule outputs are combined using the union operator.

Furthermore, Wu's representation is a special case of the CIT2 definition proposed in this paper. That is simply because non-convexity and normality, can be expressed mathematically in terms of constraints (as shown in [3]) that can then be used to generate a CAES and therefore a CIT2 FS.

Finally, we would like to mention that it is possible to build a CAES so that it includes all the ESs of an IT2 FS. In other words, given any IT2 FS, it is always possible to generate a CAES to obtain an equivalent CIT2 FS.

Specifically, given an IT2 FS $\tilde{A}$ with the FOU delimited by the upperbound and lowerbound MFs $\bar{\mu}_{\tilde{A}}$ and $\underline{\mu}_{\tilde{A}}$ we can generate the $\mathrm{CAES}_{\breve{A}}$ of the equivalent (i.e. with the same FOU) CIT2 FS $\breve{A}$ by using the conjunction of the two following constraints $C_{1}$ and $C_{2}$ : 


$$
\begin{aligned}
& C_{1}(S)= \begin{cases}\text { true } & \text { if } \mu_{S}(x)<=\bar{\mu}_{\tilde{A}}(x), \forall x \in X \\
\text { false } & \text { otherwise }\end{cases} \\
& C_{2}(S)= \begin{cases}\text { true } & \text { if } \mu_{S}(x)>=\underline{\mu}_{\tilde{A}}(x), \forall x \in X \\
\text { false } & \text { otherwise }\end{cases}
\end{aligned}
$$

However, this does not mean that there is an equivalence between the IT2 and CIT2 representations. In fact, whenever we prefer CIT2 to IT2 FSs, our goal is to work with a subset of all the ES described in the representation theorem [1] in order to keep a semantic meaning and/or consistency in data representation with the concepts we are modeling.

\section{CONCLUSION}

In this paper, we analysed the use of the concept of meaningful shapes in CT2 FSs. We showed how the current definition of CIT2 FSs that only considers as acceptable the ESs with a given shape, is sometimes not necessary to maintain a meaningful connection with the concept one is modeling, even in contexts such as human reasoning. In addition to that, the concept of meaningfulness itself has remained vague and not formally defined. To overcome these limitations, we gave a new, more general definition of CIT2 FSs based on the concept of the satisfaction of mathematical constraints to identify the shapes that are considered "meaningful" for our ESs. These constraints can be extracted, for example, by analyzing the properties that we implicitly desire when we use words (such as medium) or they can be determined so that they keep empirical or theoretical relations between the values of the universe of discourse. We also showed how the old definition can be considered as a special case of the new one and how, given an IT2 FS, is it always possible to obtain its equivalent (i.e. with the same FOU) CIT2 representation by using two constraints. Finally, we discussed the differences between our approach and the constrained approach proposed by $\mathrm{Wu}$ in [3], which can be seen as a special case of our CIT2 FSs. In future work, we will show how the new definitions presented in this paper will make the CIT2 FSs more flexible and applicable in real case scenarios which require the use of multiple (but limited) shapes for the representation of data.

\section{REFERENCES}

[1] J. M. Mendel and R. I. John, "Type-2 fuzzy sets made simple," IEEE Transactions on Fuzzy Systems, vol. 10, no. 2, pp. 117-127, Apr 2002

[2] N. N. Karnik and J. M. Mendel, "Centroid of a type-2 fuzzy set," Information Sciences, vol. 132, no. 1-4, pp. 195-220, 2001.

[3] D. Wu, "A constrained representation theorem for interval type-2 fuzzy sets using convex and normal embedded type-1 fuzzy sets, and its application to centroid computation," Proceedings of World Conference on Soft Computing, 2011.

[4] J. Aisbett, J. T. Rickard, and D. Morgenthaler, "Multivariate modeling and type-2 fuzzy sets," Fuzzy Sets and Systems, vol. 163, no. 1, pp. 78 - 95, 2011, theme: Classification and Modelling.

[5] D. Wu, H. Zhang, and J. Huang, "A constrained representation theorem for well-shaped interval type-2 fuzzy sets, and the corresponding constrained uncertainty measures," IEEE Transactions on Fuzzy Systems, pp. $1-1,2018$.
[6] J. M. Garibaldi and S. Guadarrama, "Constrained type-2 fuzzy sets," in Advances in Type-2 Fuzzy Logic Systems (T2FUZZ), 2011 IEEE Symposium on. IEEE, 2011, pp. 66-73.

[7] P. D'Alterio, J. M. Garibaldi, and A. Pourabdollah, "Exploring constrained type-2 fuzzy sets," in 2018 IEEE World Congress on Computational Intelligence (WCCI 2018), July 2018.

[8] J. M. Mendel, R. I. John, and F. Liu, "Interval type-2 fuzzy logic systems made simple," IEEE Transactions on Fuzzy Systems, vol. 14, no. 6, pp. 808-821, Dec 2006.

[9] J. M. Mendel, M. R. Rajati, and P. Sussner, "On clarifying some definitions and notations used for type-2 fuzzy sets as well as some recommended changes," Information Sciences, vol. 340-341, pp. 337 345, 2016.

[10] J. M. Mendel, "Computing with words and its relationships with fuzzistics," Information Sciences, vol. 177, no. 4, pp. 988 - 1006, 2007.

[11] A. Norwich and I. Turksen, "A model for the measurement of membership and the consequences of its empirical implementation," Fuzzy Sets and Systems, vol. 12, no. 1, pp. 1 - 25, 1984. 